\title{
A CG Generation Method of Wash Drawing
}

\author{
Shinichiro Tomohiro * Hiroki Imamura ** Hideo Kuroda * Makoto Fujimura ** \\ *Graduate School of Science and Technology, Nagasaki University \\ **Faculty of Engineering, Nagasaki university \\ \{F08314,Imamura,kuroda,makoto\}@cc.nagasaki-u.ac.jp
}

\begin{abstract}
The opportunity of process the image increases, the research on generating the image like the painting became on important in recent years. Generally individual image blog shape contents open to the public chance is increase. In the painting, the drawing in ink and wash has the feature with a framing of the outline of the Iras key of $G$ and an especially light color. Generally, it is a technique for coloring in the watercolor with a high transparency after it sketches with the pencil etc. and the brush. The outline of the image is extracted to reproduce this technique, and the stroke of the brush is reproduced. Coloring segments the area according to the inclination, and smoothes the inside.
\end{abstract}

\section{Introduction}

Recent years, sending contents privately becomes possible by spread of computers and the development of the Internet. Up to now, the enterprise etc. had delivered contents. Recently, The opportunity that the individual delivers contents through the Internet has increased. However, because the technology, the experience, and time, etc. are necessary for making contents, the generation of contents is privately difficult. Therefore, the technique for easily generating contents is hoped for. The computer graphics (CG) became one of the important contents.

CG chiefly divides into a photo real and non-photo real as an expression. A photo real is an expression technique for bringing it close to the more photograph taken from life with real CG as shown in Figure 1. Non-photo real is $\mathrm{CG}$ of a hand-written style shown Figure 2. There are two generation techniques of $\mathrm{CG}$ in non-photo real. One is a method which draws by human using the paint software. Another is a technique for generating by computers autonatically. The techniques of oil painting style CG [1] and the watercolor style CG[2], etc. are proposed as an expression of $\mathrm{CG}$ of non-photo real by computers automatically .
We focus on the feature of wash drawing and aim at the proposal of wash drawing $\mathrm{CG}$ generation technique

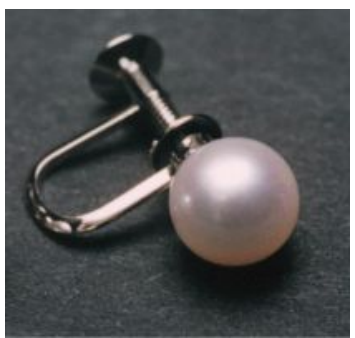

Fig 1: Photo real CG

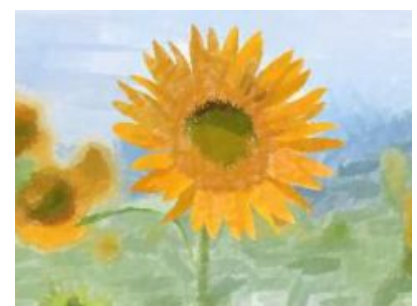

Fig 2: Non-photo real CG

\section{Feature of wash drawing}

To aim at the generation of wash drawing $\mathrm{CG}$ in this research, the feature of the wash drawing is considered. The wash drawing is a technique for putting blight, peculiar colors after sketching and the outline is drawn[3]. Drawing of the wash drawing and is done in general as follows. First, the object is sketched with the pencil etc. The sketch draws the outline, and the shadow is not applied. Painting materials used to sketch are with a pencils and brush, and not chiefly so blotted. Next, it colors. Coloring lightly puts blight, peculiar colors. However, because the transparency is important, the color mixture and the piling paint are reduced. This is because the colors dulled when a lot of paints are mixed. Moreover, the place not to be painted either exists. Paints used to paint are watercolor with high transparency. The outline uses the pencil and the brush. Paints use the watercolor with a high transparency. Because the watercolor and wash drawing use the same paints.Those methods have the same features.

Figures 3 and 4 show the example of the wash drawing. Figures 5 and 6 show the example of the watercolor. The feature of the wash drawing and the watercolor is summarized in Table 1. 
Table 1 :Comparison between wash drawing and wash with watercolor

\begin{tabular}{|c|c|c|}
\hline & drawing in ink & watercolor \\
\hline $\begin{array}{c}\text { Outline } \\
\text { feature }\end{array}$ & Framing. & Not framing. \\
\hline Vividness & Subtle colors & Normal colors \\
\hline brightness & Bright & Bright or dark \\
\hline
\end{tabular}

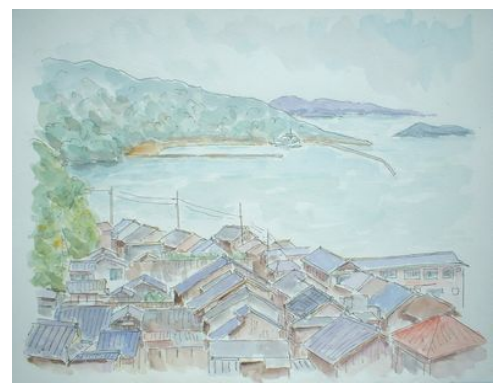

Fig 1: Example of wash drawing \#1

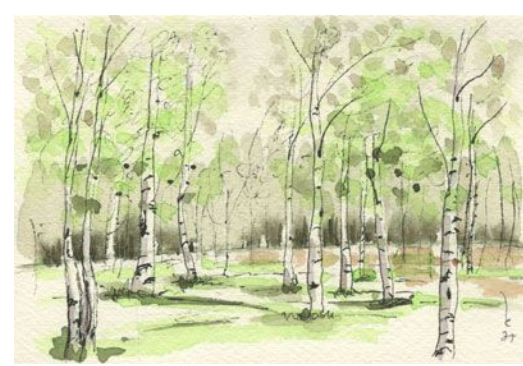

Fig 2: Example of wash drawing \#2

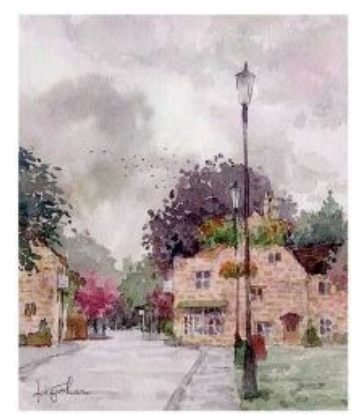

Fig 4: Example of water color \#1

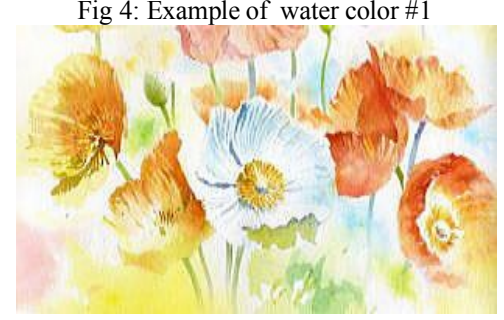

Fig 5 :Example of water color \#2

\section{Proposal technique}

The wash drawing is a technique for drawing clear outlines, and putting blight, peculiar colors on that. Therefore, the proposal technique divides into the drawing processing and the coloring processing in the outline. Figure 7 shows the flow chart of the proposal technique.

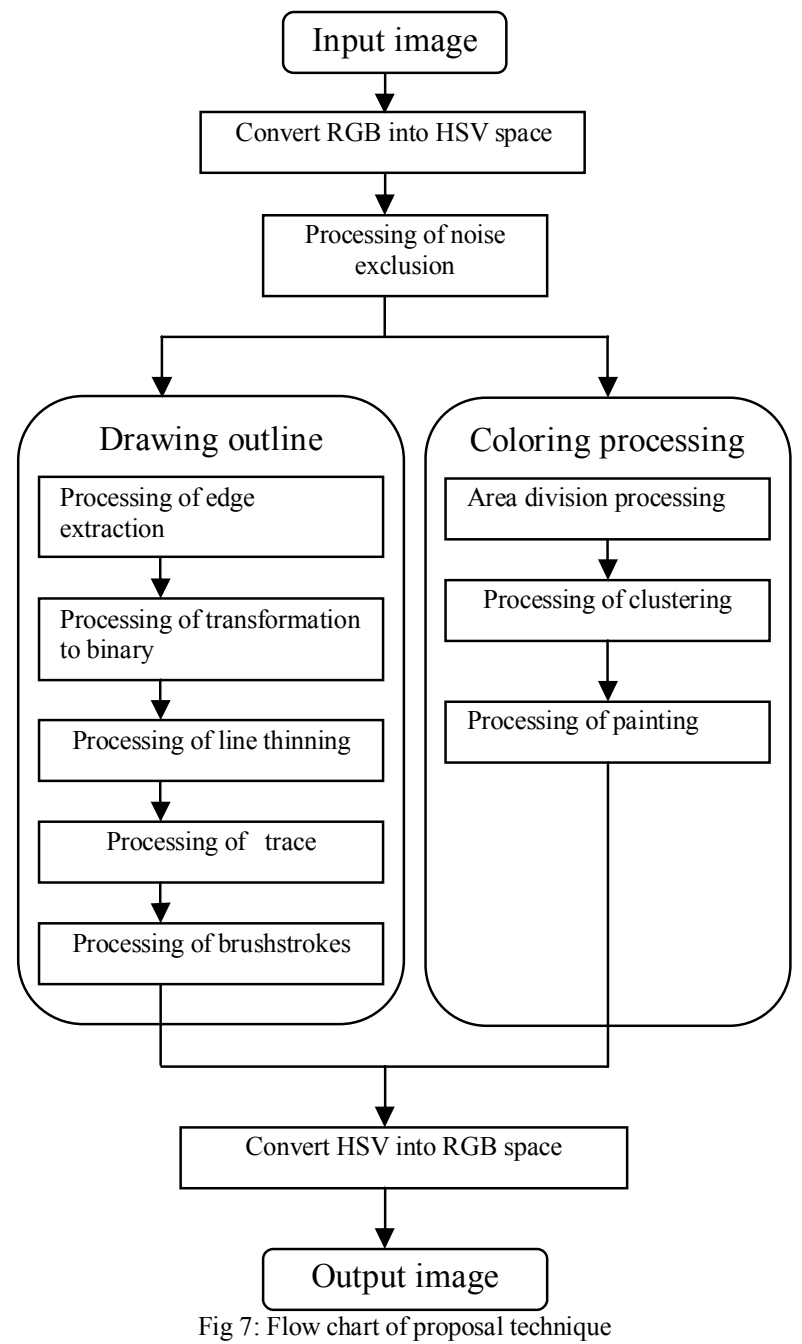

\subsection{Preprocessing}

In this section, we show the preprocessing before the drawing processing and the coloring processing in the outline. 


\subsubsection{Conversion RGB into HSV space}

In general, the image data uses RGB space. RGB space expresses the color by three parameters, in a word, $\operatorname{red}(\mathrm{R})$, green $(\mathrm{G})$, and blue(B). When the color brightness, and vividness are changed, it is necessary to change all of the three parameters. So we convert RGB into the HSV space. The HSV space expresses the color by the parameter of hue $(\mathrm{H})$, the saturation $(\mathrm{S})$, and value $(\mathrm{V})$. There is an advantage that we can change colors intuitively.

\subsubsection{Noise exclusion}

Because the noise has mixed with the input image, we exclude noise. The purpose is to undertake a big influence when the noise mixes to the edge extraction processing. We use general median filter for noise exclusion.

\subsection{Drawing outline}

We show explains processing that expresses the outline of the wash drawing. The profile line is extracted to express the outline of the wash drawing, and the outlines are drawn while tracing it.

\subsubsection{Processing of edge extraction}

To extract the outline of the image, we execute edge extraction. The first derivation is taken for pixel $f(j, k)$ that includes the input pixel[4]. The first derivation is squared to have to extract the outline clearly and the outline is extracted clearly. $X$ regularizes it so that the maximum value may become 255 . Because the difference of the place that it is an outline and is not the outline become large, the outline can be extracted more clearly. The expression of the edge extraction is expressed equation (1) and regularization is expressed equation (2). The output image that regularizes the input image in $f(j, k)$ is expressed as the $X(j, k)$.

$$
\begin{aligned}
g_{j, k} & =\frac{1}{\mathbf{8}} \sum_{i=j-1}^{j+1} \sum_{t=k-\mathbf{1}}^{k+1}\left(f_{j, k}-f_{i, t}\right)^{2} \\
X_{j, k} & =\frac{g_{j, k} \times \mathbf{2 5 5}}{\operatorname{MAX}\left(g_{j, k}\right)}
\end{aligned}
$$

\subsubsection{Processing of transformation binary}

We set the values of the image intensity as 1 , if the value of the image intensity is larger than threshold Th, on the other hand, we set that as 0 , if that is smaller than
Th. Because the appropriate threshold of Th is different in the part of the image, we decide the appropriate threshold of Th based on standard divition expressed by (Equation (4)), where (Equation (3)-(7)).

$$
\begin{aligned}
E_{x, y} & =\frac{\mathbf{1}}{\mathbf{5} \times \mathbf{5}} \sum_{j=x-4}^{x+\mathbf{4}} \sum_{k=y-4}^{y+\mathbf{4}} X_{j, k} \\
V_{x, y} & =\frac{\mathbf{1}}{\mathbf{5} \times \mathbf{5}} \sum_{j=x-4}^{x+4} \sum_{k=y-4}^{y+\mathbf{4}}\left(E_{x, y}-X_{j, k}\right) \\
\sigma_{x, y} & =\sqrt{V_{x, y}} \\
E d_{x, y} & =\sigma_{x, y}+\mathbf{1} \\
X_{x, y} & =\left\{\begin{array}{l}
\mathbf{0}\left(X_{x, y}<E d_{x, y}\right) \\
\mathbf{1}\left(X_{x, y}<E d_{x, y}\right)
\end{array}\right.
\end{aligned}
$$

\subsubsection{Line thinning processing}

Because the width of the line is bold, and outline drawing in lines position cannot be uniquely specified on the outlines. Then, it is necessary to process the line thinning that thinly adjusts the stroke width to one. The line thinning is removes a corresponding one to the operator in Figure 8[5][6].

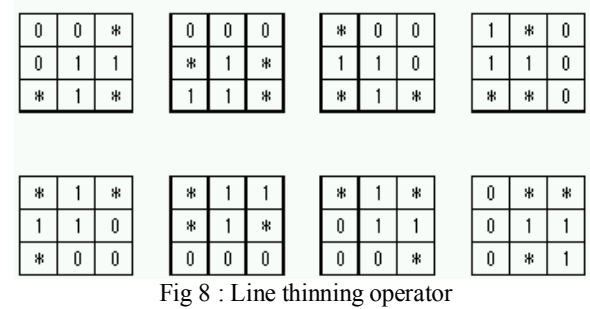

\subsubsection{Processing of trace}

After processing of the line thinning, irregularities of lines are generated. To smooth the lines, we execute the following processing. First we trace the thing lines, then make node, connect each node. We set the interval of the node as 10 pixels.

\subsubsection{Processing of brushstrokes}

In case of only connecting the nodes, the connected of lines are generated. Therefore, we make it smooth by interpolation of nodes. We use figure 10 method for interpolation of nodes. Then we trace the smoothed line by blush models shown in Figure 11. In this process, we first use thin blush model, then we use bolder blush models. As an example, we show the input image and processed image in figure 12 and figure 13 respectively. 


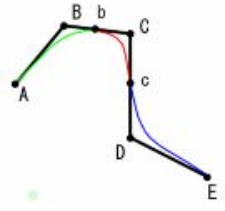

Fig 10: Interpolation of nodes (capital letters are node)

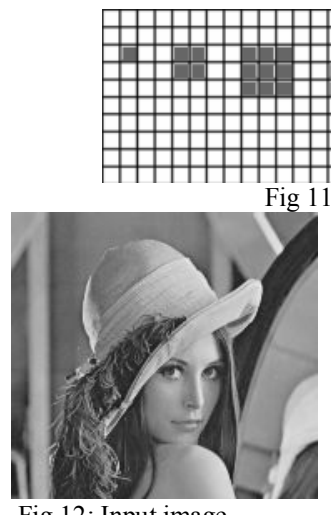

Fig 12: Input image
Fig $11:$ Blush models

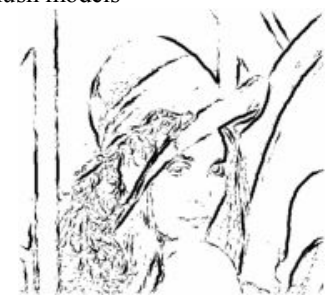

Fig 13: Drawing outline image

\subsection{Coloring processing}

\subsubsection{Area division processing}

To extract flow of the stroke, we segment image in gradient of image intensities(figure 13, figure 14).The expression of the gradient of image is shown in equation (8)-(11).

$$
\begin{aligned}
& x_{-} \operatorname{par}_{j, k}=\left(X_{j+\mathbf{1}, k+1}+X_{j+\mathbf{1}, k}+X_{j+\mathbf{1 , k - 1}}\right) \\
& -\left(X_{j-1, k+1}+X_{j-1, k}+X_{j-1, k-1}\right) \\
& y_{-} \operatorname{par}_{j, k}=\left(X_{j+1, k+1}+X_{j, k+1}+X_{j-1, k+1}\right) \\
& -\left(X_{j+1, k-1}+X_{j, k-1}+X_{j-1, k-1}\right)
\end{aligned}
$$

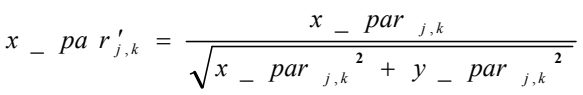

$$
\begin{aligned}
& y_{-} p a r_{j, k}^{\prime}=\frac{y_{-p a r_{j, k}}}{\sqrt{x-p a r_{j, k}^{2}+y_{-}{ }^{2} a r_{j, k}^{2}}} \\
& R_{j, k}=\arctan \left(\frac{y-p_{j, k}}{x-p_{j, k}}\right)
\end{aligned}
$$

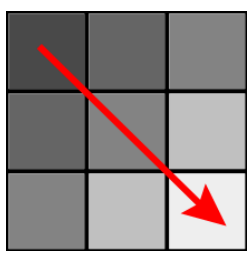

Fig 13: Image of gradient of image intensities

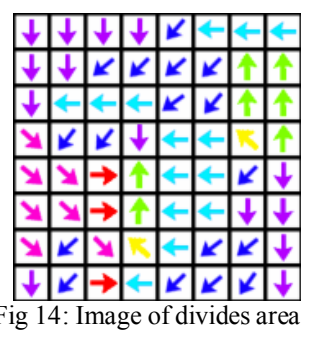

\subsubsection{Processing of clustering}

To integrate isolated segmented regions, we integrate isolated segmented regions, we integrate the segmented regions in each 30 degree. By this process, we can integrate isolated regions (figure 14) larger segmented regions (figure 15-16).we execute this process against value element in $\mathrm{HSV}$ and saturation element in HSV respectively. The example of segmentation results are shown in figure 17 and figure 18 respectively.

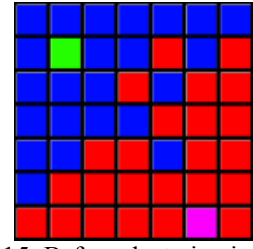

Fig 15: Before clustering image
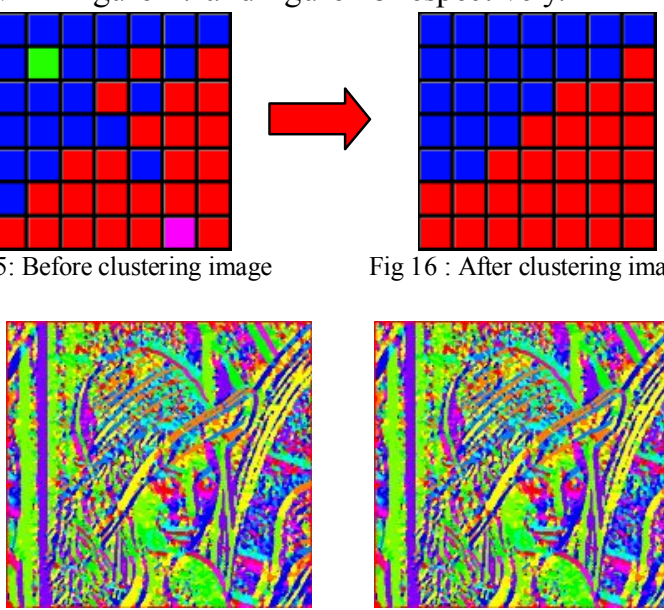

Fig17: Value element clustering Fig18: Saturation element clustering

\subsubsection{Processing of painting}

In this process, we paint each segmented regions by equation (19) and equation (20) where S_ave and $\mathrm{H} \_$ave denote the mean of saturation and Hue.

$$
\begin{aligned}
V i_{j, k} & =V \text { _ave } \times \mathbf{1 . 5} \\
S i_{j, k} & =\left\{\begin{array}{c}
\left.S_{-} \text {ave } \times \mathbf{0 . 4} \text { (if } S_{j, k}<S_{-} \text {ave }\right) \\
\left.S_{-} \text {ave } \times \mathbf{0 . 6} \quad \text { (if } s \geq S_{-} \text {ave }\right)
\end{array}\right.
\end{aligned}
$$




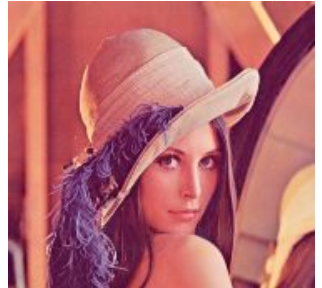

Fig 19: Input image

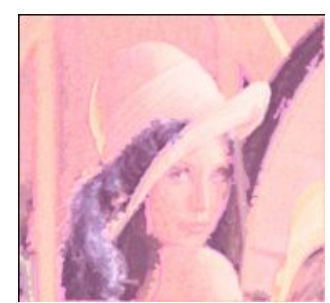

Fig 20 : Coloring processing image

\subsection{Output image}

We generated the output image by integration of stroke image (figure 13) and painted image (figure 20). As an example, we show an input image and its output image in figure 21 and figure 22 respectively.

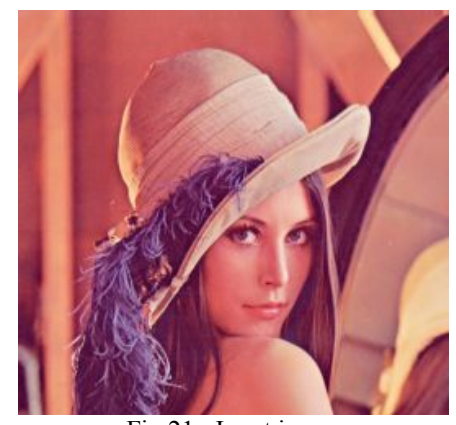

Fig 21 : Input image

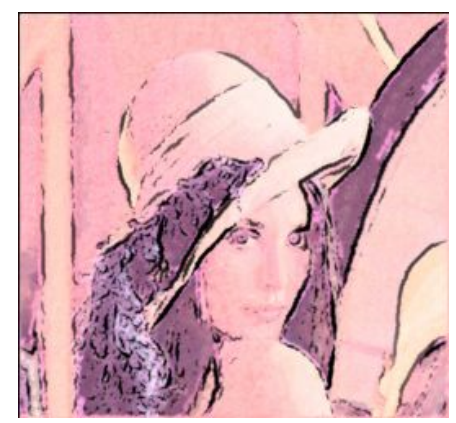

Fig 22: Output image

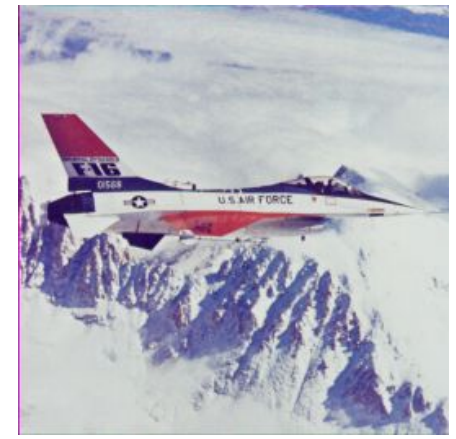

Fig 23: Input image

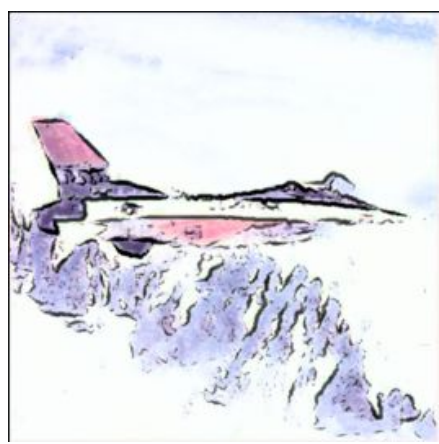

Fig 24: Output image

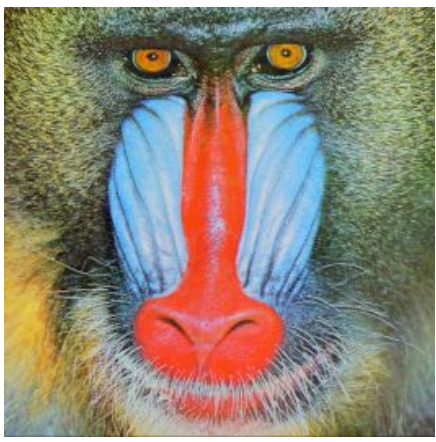

Fig 25: Input image

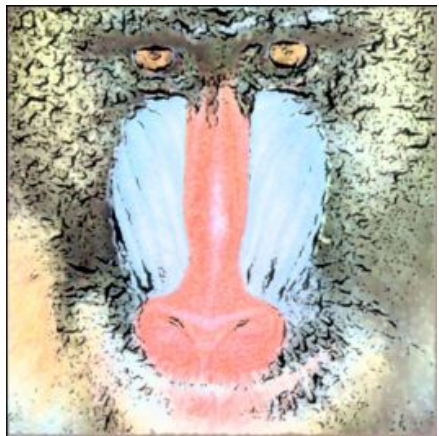

Fig 26 : Output image

\section{Experiment result}

To evaluated the proposed technique, we executed the subjective experiment. We should input images and output image by the proposed technique to 10 student, and made them evaluate by questionnaires as follows.

1. The profile line looks smooth.

2. The places in the outline do not shift.

3. It seems to be painted by brush.

4. The color on the output image is brighter.

5. The color on the output image is lighter.

6. The color on the output image is lightened.

7. The output image can express the blush model. 
Table 2: Result of evaluation experiment

\begin{tabular}{|c|c|c|c|c|c|c|c|c|}
\hline Evaluation & 1 & 2 & 3 & 4 & 5 & Average & $\begin{array}{c}\text { Decentraliz } \\
\text { ation }\end{array}$ & $\begin{array}{c}\text { Standard } \\
\text { deviation }\end{array}$ \\
\hline Item 1 & 0 & 0 & 0 & 4 & 6 & 4.6 & 0.26 & 0.51 \\
\hline Item 2 & 0 & 0 & 0 & 6 & 4 & 4.4 & 0.26 & 0.51 \\
\hline Item 3 & 1 & 0 & 1 & 6 & 2 & 3.8 & 1.28 & 1.13 \\
\hline Item 4 & 0 & 0 & 0 & 3 & 7 & 4.7 & 0.23 & 0.48 \\
\hline Item 5 & 0 & 1 & 0 & 6 & 3 & 4.1 & 0.76 & 0.87 \\
\hline Item 6 & 0 & 0 & 2 & 4 & 4 & 3.9 & 1.65 & 1.28 \\
\hline Item 7 & 0 & 0 & 1 & 8 & 1 & 4.0 & 0.22 & 0.48 \\
\hline
\end{tabular}

The result of the questionnaires is shown in table 2. In each item, we obtained high score. However, in item 3, we obtained score 1.This is because the blush model is very simple. Therefore, we intend to improve the blush model.

\section{Conclusion}

To generate CG like wash drawing, we proposed a CG generation method for wash drawing. To evaluate the proposed technique, we execute the subjective experiment. From the results of the experiments, we obtained high scores in each items of questionnaires.

\section{Reference}

[1] "Generation of watercolor style image that considers drawing technique"

Http://nislab.is.s.u-tokyo.ac.jp/nis/cdrom/pg/watercolor JSAS 2004.pdf

[2] "Real-time generation and retrieval of no photo real image"

Http://www.taf.or.jp/publication/kjosei 21/pdf/p458.pdf [3] "Real-time generation and retrieval of no photo real image"

Http://www.taf.or.jp/publication/kjosei 21/pdf/p458.pdf [4] Koichi Sakai "digital image processing introduction "2005 corona company

[5] "Line thinning processing script model"

Http://www.esrij.com/support/erdas/faq/thinning/thinnin g.jsp

[6] " Hilditch's Algorithm for Skeletonization" http://jeff.cs.mcgill.ca/godfried/teaching/projects97/azar/ skeleton.html 